\title{
Nonlinear Dynamics and Spectral Stability of Optoelectronic Microwave Oscillators
}

\author{
Yanne Kouomou Chembo, Laurent Larger and Pere Colet
}

\begin{abstract}
We use a nonlinear dynamics approach to study the deterministic behavior of ultra-pure microwave generators referred to as optoelectronic oscillators. In conventional studies, the standard nonlinear effects are very strongly rejected because they generate harmonics of the microwave frequency that are definitely out of the selective oscillator bandwidth. However, we show that the nonlinearity still affects the slowly varying dynamics of microwave envelope, thereby inducing dynamical instabilities within the oscillator bandwidth. Starting from a full integro-differential model, we use the multiple timescales method to build a delay-differential equation for the slowly-varying complex envelope of the microwave. Then, the corresponding stationary solutions are derived, and the stability of their amplitude and phase is investigated in detail as a function of the feedback gain. We evidence essential bifurcation phenomena, and in particular we demonstrate that the generated microwave may turn unstable if the gain is increased beyond a precise critical value. This nonlinear dynamics approach therefore demonstrates that the ultra-pure microwave's amplitude does not monotonously increase with the gain. The theoretical study is confirmed by numerical simulations and experimental measurements.
\end{abstract}

Keywords-Optoelectronic devices, microwave generation, delay effects, nonlinear oscillators.

\section{INTRODUCTION}

A very efficient way to produce ultra-pure microwaves has been proposed few years ago by Yao and Maleki [1], [2], and it is known under the name of optoelectronic oscillator (OEO). The principle of operation of this oscillator relies on an innovative energy storage principle, based on very long, low losses optical fiber delay lines, instead of the classical concept of high-finesse resonators.

The most basic architecture of the OEO consists in a Mach-Zehnder (MZ) modulator which modulates nonlinearly through its $\sin ^{2}$ interference transfer function a continuous wave coherent light beam. The radio-frequency (RF) modulated optical output then circulates within an extremely long fiber delay-line, whose typical length is few kilometers. This optical signal is afterwards converted back into an electrical signal which is amplified, narrowly filtered around the microwave frequency to be generated, and connected to the electrode of the Mach-Zehnder. Typically, the central frequency of the filter is between 1 and $40 \mathrm{GHz}$, while the bandwidth of the RF filter has few tens of $\mathrm{MHz}$. This closed-loop architecture therefore plays the role of an

Y. Kouomou Chembo (email: yanne.chembo@femto-st.fr) and L. Larger are with the Optics Department, FEMTO-ST Institute, 16 Route du Gray, 25030 Besançon cedex, France.

$\mathrm{P}$. Colet is with the Instituto de Física Interdisciplinar y Sistemas Complejos IFISC (CSIC-UIB), E-07122 Palma de Mallorca, Spain.

Y.K.C. acknowledges a research fellowship from the Région de Franche-Comté in France. Financial support from MEC (Spain) and FEDER under projects TEC2006-10009 (PhoDeCC) and FIS200760327 (FISICOS) is acknowledged. optoelectronic ring cavity, but however, its quality factor is very low, typically between 100 and 40000. In fact, the outstanding performances of this oscillator originate from the few-kilometers long delay line, which generates thousands of narrowly spaced cavity modes. The long delay line thus plays the role of a strongly phase shifting element, thereby resulting in a high quality factor in terms of phase versus frequency selectivity. The spectral purity of the oscillation is then mainly determined by the ring cavity length and by the microwave frequency, rather than by the gain profile of the medium (which is in fact the amplitude selectivity of the filter) [1]. Even though hundreds of theses microwave cavity modes do fit within the bandwidth of the RF filter, only one mode is amplified under optimal conditions, and oscillates in a continuous, stable way, typically through a selective mode competition process.

As earlier noticed, the purity of the microwaves generated with OEOs has been found to be very superior to the previous state of the art resonator-based oscillators. These performances can even be improved if some modifications are brought to the original single-loop architecture. For example, the spurious cavity-modes can be overdamped by using the Vernier effect of a dual-loop architecture [3], or alternatively, by designing a suitable coupling between two OEOs [4]. It is also possible to build tuneable OEOs [5], or even to enhance the frequency selection using a photonic filter instead of a RF filter [6]. Therefore, owing to this multiplicity and flexibility of architectures, OEOs are expected to find applications for time-frequency metrology, and of course for the next generation of high-precision radars/sensors, where extreme purity for the reference microwave is crucial. OEOs are also of great interest for many applications in spatial and lightwave technologies. Just taking few examples in the latter case, OEOs have been pointed out as adequate systems to produce ultra-low-jitter optical pulses at high rates $(>10 \mathrm{GHz})$, to produce multiwavelength and synchronized optical pulses, or to perform clock recovery at bit rates as high as $40 \mathrm{~Gb} / \mathrm{s}$ (see ref. [7] and references therein).

Despite this large amount of experimental investigations developed around the concept of OEO, very few has been done to investigate theoretically its dynamical properties. This lack of analytical insight is due to the extreme features of this system: very large delayed feedback, widely spaced timescales, and potentially strong nonlinearity induced by a multiple-valued (non-monotonous) nonlinear transfer function. These difficulties explain why most of OEO studies do implicitly assume no time-dependent amplitude dynamics, as they try to capture essentially sta- 
tionary features.

However, the stationary hypothesis may not be always satisfied. A priori, it can logically be considered that OEOs may as any delayed dynamical system display other behaviors. In fact, the OEO belongs to the wide family of electro-optical systems with delayed feedback, which was first introduced by Neyer and Voges back to the early 80 s [8]. Later, many studies had been devoted to this class of systems, evidencing interesting nonlinear behaviors such as bistability, hyperchaos, and even chaotic breathers (see refs. [9], [10] and references therein). In particular, one of the strongest research line associated to this architecture is still optical chaos cryptography [11], [12], [13].

It therefore appears that as far as the OEO is concerned, absence of a dynamical framework prevents from a deep understanding of existing architecture's performances, and of course does not enable the prediction of eventual instabilities. The main objective of the paper is then the explicit derivation of a delay-differential equation which can describe accurately the deterministic dynamical behavior of OEOs, as well as a detailed stability study of the solutions of interest. These investigations therefore complement earlier results which have recently been presented in ref. [14].

The plan of the article is the following. In Sec. II, we present the experimental set-up corresponding to the single-loop OEO under study. The modeling of the OEO's dynamics is performed in Sec. III. We will use the multiple time scales method to derive an equation for the complex envelope of the microwave. Section IV is devoted to the existence and stability of the stationary solutions. A detailed analysis is carried out for the amplitude and for the phase of the microwave output. These results are discussed in Sec. VII, and are completed in Secs. V and VI by numerical simulations and experimental measurements respectively. We end with some concluding remarks in the final section.

\section{THE SYSTEM}

A shematic representation of the OEO under study is displayed in Fig. 1, and the oscillation loop consists of the following elements:

- A wideband integrated optics $\mathrm{LiNbO}_{3}$ Mach-Zehnder modulator; it is seeded by a continuous-wave semiconductor laser of optical power $P$, which serves as a bifurcation parameter for scanning the OEO feedback gain; the modulator is characterized by a half-wave voltage $V_{\pi_{D C}} \simeq$ $V_{\pi_{R F}}=4.2 \mathrm{~V}$, which defines the amplitude scale required at the microwave Mach-Zehnder driving voltage $V(t)$ for operation in the nonlinear regime.

- A thermalized $4 \mathrm{~km}$ fiber performs a time delay of $T=20$ $\mu$ s on the microwave signal carried by the optical beam; the long delay is intended to support thousands of the microwave ring-cavity modes, whose free spectral range is equal to $\Omega_{T} / 2 \pi=1 / T=50 \mathrm{kHz}$.

- A fast amplified photodiode with a conversion factor $S=$ $2.2 \mathrm{~V} / \mathrm{mW}$.

- A narrow-band microwave radio-frequency (RF) filter, intended to select the frequency range for the amplified modes; its central frequency is $\Omega_{0} / 2 \pi=3 \mathrm{GHz}$, and the
$-3 \mathrm{~dB}$ bandwidth is $\Delta \Omega / 2 \pi=20 \mathrm{MHz}$.

- A microwave amplifier with gain $G$ is required to close the loop. All optical and electrical losses are gathered in a single attenuation factor $\kappa$.

It appears to some extent that the global features of the electro-optical systems which are generally studied in the literature are qualitatively at the extreme opposite of those of the OEO: on the one the hand, we have a wide bandwidth and a relatively short delay-line, intended to generate a chaotic radio-frequency spectrum as flat and as broad as possible [9], [11], [12]; on the other hand, we have a narrow bandwidth and a very long fiber cavity in order to select a single cavity mode, that is, a single frequency. For this latter case, high gains are more difficult to obtain because of the high losses induced by the narrowband RF filter, and low gain obtained from the low noise photodiodes required to ensure the lowest phase noise level performance.

\section{THE MODEL}

\section{A. Equation for the instantaneous microwave amplitude}

The narrowband filter has a central frequency $\Omega_{0}$ and a bandwidth $\Delta \Omega$. For the sake of simplicity, we will assume that it is a first order linear band-pass filter, so that the dynamics of the system will be ruled on the one hand by the integro-differential relation between its input and its output, and on the other hand by the closure condition imposed by the feedback loop.

Effectively, as it is known from the theory of linear filters, the input and output of a band-pass filter are related as

$V_{\text {out }}(t)+\frac{1}{\Delta \Omega} \frac{d}{d t} V_{\text {out }}(t)+\frac{\Omega_{0}^{2}}{\Delta \Omega} \int_{t_{0}}^{t} V_{\text {out }}(s) d s=V_{\text {in }}(t)$.

On the other hand, $V_{i n}(t)$ is also the output voltage of the photodetector; hence, it is proportional to the optical power of the semiconductor laser which had been electrooptically modulated at the time $t-T$ by the amplified voltage $V=\kappa G V_{\text {out }}$. Consequently we have the relationship

$$
V_{i n}(t)=S P \cos ^{2}\left[\frac{\pi V(t-T)}{2 V_{\pi_{R F}}}+\frac{\pi V_{B}}{2 V_{\pi_{D C}}}\right] .
$$

Therefore, the radio-frequency input voltage $V(t)$ of the Mach-Zehnder modulator obeys the following equation

$$
\begin{aligned}
& V(t)+\frac{1}{\Delta \Omega} \frac{d}{d t} V(t)+\frac{\Omega_{0}^{2}}{\Delta \Omega} \int_{t_{0}}^{t} V(s) d s \\
= & \kappa G S P \cos ^{2}\left[\frac{\pi V(t-T)}{2 V_{\pi_{R F}}}+\frac{\pi V_{B}}{2 V_{\pi_{D C}}}\right] .
\end{aligned}
$$

This equation can be rewritten under this simplified and dimensionless form

$$
x+\tau \frac{d x}{d t}+\frac{1}{\theta} \int_{t_{0}}^{t} x(s) d s=\beta \cos ^{2}[x(t-T)+\phi],
$$

where $x(t)=\pi V(t) / 2 V_{\pi_{R F}}$ is the dimensionless voltage at the input of the Mach-Zehnder, $\beta=\pi \kappa S G P / 2 V_{\pi_{R F}}$ is the 


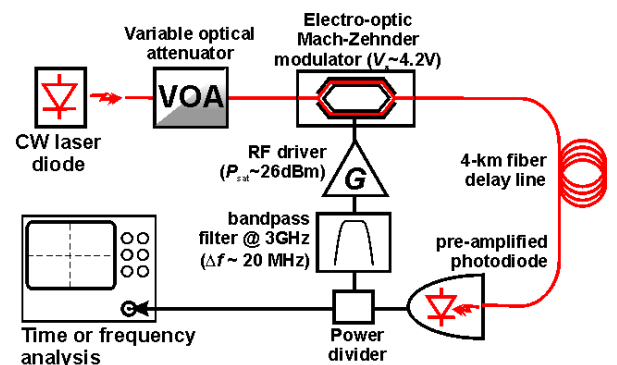

Fig. 1. Experimental setup for the single-loop OEO.

normalized feedback gain, $\phi=\pi V_{B} / 2 V_{\pi_{D C}}$ is the MachZehnder off-set phase, while $\tau=1 / \Delta \Omega$ and $\theta=\Delta \Omega / \Omega_{0}^{2}$ are the characteristic timescale parameters of the bandpass filter.

The dynamics of the OEO can unfortunately not be studied under the form of Eq. (4), because the splitting between the fastest and slowest dynamical timescales is too high [14]. However, owing to the bandpass filter, only the oscillations whose frequency is within the bandwidth can arise. Therefore, an option to circumvent this problem is to represent the solution under the form of a carrier of frequency $\Omega_{0}$, slowly modulated by a complex amplitude $\mathcal{A}(t)$, yielding

$$
x(t)=\frac{1}{2} \mathcal{A}(t) e^{i \Omega_{0} t}+\frac{1}{2} \mathcal{A}^{*}(t) e^{-i \Omega_{0} t}
$$

with $\mathcal{A}=|\mathcal{A}| e^{i \psi}$. In the next section, we derive an equation for the complex envelope amplitude $\mathcal{A}(t)$, which is assumed to vary slowly relatively to the central carrier frequency $\Omega_{0}$, that is $|\dot{\mathcal{A}}(t)| \ll \Omega_{0}|\mathcal{A}(t)|$.

Generally, the frequency-domain approach is privileged in the theoretical studies of microwave generators. This is due to the fact that resonator-based oscillators have an extremely high quality factor $Q\left(>10^{5}\right)$, inducing extremely slow energy decay-time compared to the fast oscillation period. These huge timescale differences are generally incompatible with affordable numerical integration and tractable analytical developments in the time domain. However, in the case of the OEO, we should be able to keep the time-domain description because of the relatively low quality factor of the microwave resonant filter (here, $\left.Q=\Omega_{0} / \Delta \Omega=150\right)$.

\section{B. Equation for the microwave envelope}

After time derivation, Eq. (4) can be rewritten under the form

$$
\frac{d^{2} x}{d t^{2}}+\Omega_{0}^{2} x=\epsilon \Omega_{0} \frac{d}{d t}\left\{-x+\beta \cos ^{2}[x(t-T)+\phi]\right\},
$$

where

$$
\epsilon=\frac{\Delta \Omega}{\Omega_{0}}=\frac{1}{Q}
$$

is the smallness parameter.
There are three different timescales in the system, the carrier frequency $\Omega_{0} / 2 \pi$ in the $\mathrm{GHz}$ range, the filter halfbandwidth $\mu / 2 \pi$ in the $\mathrm{MHz}$ range, and at last the free spectral range $\Omega_{T} / 2 \pi$ in the $\mathrm{kHz}$ range. The separation of timescales by the same 3 orders of magnitudes makes the OEO an ideal case for the multiple time scales method [15], [16]. This method relies on the expansion of the variables in function of the various timescales involved in the system. Then, a different dynamics is assumed at each timescale, so that an evolution equation is derived at each corresponding timescale.

Let us then consider the smallness parameter $\epsilon$ which separates the various timescales as $T_{k}=\epsilon^{k} t$, where $k$ is a positive integer. According to this definition, $T_{0}=t$ is the fastest timescale, while as $k$ increases, the times $T_{k}$ are attached to slower variations. However, only a limited number of timescales is needed to take into account all the dynamical features of the system: here, the dynamics of interest can be tracked with a first order expansion of the variable $x(t)$ following

$$
x(t)=x_{0}\left(T_{0}, T_{1}\right)+\epsilon x_{1}\left(T_{0}, T_{1}\right) .
$$

On the other hand, the different timescales are considered independent, so that the time derivatives expand as $d / d t=$ $D_{0}+\epsilon D_{1}$ and $d^{2} / d t^{2}=D_{0}^{2}+2 \epsilon D_{0} D_{1}$, with $D_{k} \equiv \partial / \partial T_{k}$. We are therefore restricted here to two timescales: a fast timescale $T_{0}$, and a slow timescale $T_{1}$.

After introducing these expanded terms into Eq. (6), it is found that the timescale components $x_{0}$ and $x_{1}$ obey the following equations at the various orders of $\epsilon$

Order $\epsilon^{0}$

$$
D_{0}^{2} x_{0}+\Omega_{0}^{2} x_{0}=0
$$

Order $\epsilon^{1}$

$$
\begin{aligned}
& D_{0}^{2} x_{1}+\Omega_{0}^{2} x_{1}=-2 D_{0} D_{1} x_{0} \\
& +\Omega_{0} D_{0}\left\{-x_{0}+\beta \cos ^{2}\left[x_{0}\left(T_{0}-T\right)+\phi\right]\right\} .
\end{aligned}
$$

From Eq. (9), the solution at order $\epsilon^{0}$ is given by

$$
x_{0}\left(T_{0}, T_{1}\right)=\frac{1}{2} \mathcal{A}\left(T_{1}\right) e^{i \Omega_{0} T_{0}}+\frac{1}{2} \mathcal{A}^{*}\left(T_{1}\right) e^{-i \Omega_{0} T_{0}},
$$

corresponding to a fast quasi-sinusoidal oscillation at frequency $\Omega_{0}$, whose amplitude may eventually vary at the slow timescale $T_{1}$.

As far as Eq. (10) is concerned, a divergence of $x_{1}$ to infinity can only be prevented by canceling the resonant terms in $\Omega_{0}$ in the right-hand side. In order to obtain this solvability condition, we first use the Jacobi-Anger expansion

$$
e^{i z \cos \alpha}=\sum_{n=-\infty}^{+\infty} i^{n} \mathrm{~J}_{\mathrm{n}}(z) e^{i n \alpha}
$$

where $\mathrm{J}_{\mathrm{n}}$ is the $n$-th order Bessel function of the first kind. The nonlinear $\cos ^{2}$ term associated with the electro-optical 
modulation can be expanded in harmonics of $\Omega_{0}$ following $\cos ^{2}\left[x_{0}\left(T_{0}-T\right)+\phi\right]=\mathcal{C}_{0}+\sum_{n=1}^{+\infty}\left\{\frac{1}{2} \mathcal{C}_{n} e^{i n \Omega_{0} T_{0}}+\right.$ c.c. $\}$

where c.c. stands for the complex conjugate of the preceding term, and

$$
\begin{aligned}
\mathcal{C}_{0}= & \frac{1}{2}+\frac{1}{2} \mathrm{~J}_{0}\left[2\left|\mathcal{A}\left(T_{1}-\epsilon T\right)\right|\right] \cos 2 \phi \\
\mathcal{C}_{n}= & \frac{1}{2} \mathrm{~J}_{\mathrm{n}}\left[2\left|\mathcal{A}\left(T_{1}-\epsilon T\right)\right|\right] i^{n} e^{i n \psi\left(T_{1}-\epsilon T\right)} e^{-i n \Omega_{0} T} \\
& \times\left\{e^{2 i \phi}+(-1)^{n} e^{-2 i \phi}\right\}, \quad n \neq 0 .
\end{aligned}
$$

Using Eqs. (11) and (13), the solvability condition of Eq. (10) can be expressed as

$$
-\frac{\partial \mathcal{A}}{\partial T_{1}}+\frac{1}{2} \Omega_{0}\left\{-\mathcal{A}+\beta \mathcal{C}_{1}\right\}=0
$$

which is also sometimes referred to as a secular equation. It may be interesting to note that owing to the narrow-band $\mathrm{RF}$ filter, only the resonant term $\mathcal{C}_{1}$ is sustained and has an influence in the system's dynamics. All other spectral terms are far outside the bandwidth of the system, and they are so strongly damped that they do not have any noticeable effect.

Using the relationship $T_{1}=\epsilon t$, Eq. (15) can be rewritten under the following final form after some straightforward algebra

$$
\dot{\mathcal{A}}=-\mu \mathcal{A}-2 \mu \gamma e^{-i \sigma} \mathrm{Jc}_{1}\left[2\left|\mathcal{A}_{T}\right|\right] \mathcal{A}_{T},
$$

where $\mu=\Delta \Omega / 2$ is the half-bandwidth of the radiofrequency filter, $\sigma=\Omega_{0} T$ is the round-trip phase shift of the microwave, $\gamma=\beta \sin 2 \phi$ is the effective gain of the feedback loop, and $\mathrm{Jc}_{1}$ is the Bessel-cardinal function defined as $\mathrm{Jc}_{1}(x)=\mathrm{J}_{1}(x) / x$. The overdot denotes here time derivation relatively to the time $t$, and we have adopted the notation $\mathcal{A}_{T} \equiv \mathcal{A}(t-T)$.

Equation (16) can furthermore be simplified. Effectively, the term $e^{-i \sigma}$ is due to the fact that the central frequency $\Omega_{0}$ of the RF filter may not coincide with the true frequency $\Omega_{c}$ of the carrier: it is therefore a detuning parameter, which can be set to \pm 1 by an adequate choice of the frequency reference. In the next section we will demonstrate that it is appropriate to set $e^{-i \sigma}=-1$, that is, $\sigma=\pi \bmod$ $[2 \pi]$. Hence, introducing the detuning frequency $\varpi$ such that

$$
\varpi T=\sigma \bmod [2 \pi]
$$

we may use the phase-shift $\mathcal{A} \rightarrow \mathcal{A} e^{i(\sigma+\pi)}$ to transform Eq. (16) into

$$
\dot{\mathcal{A}}=-\mu \mathcal{A}+2 \mu \gamma \mathrm{Jc}_{1}\left[2\left|\mathcal{A}_{T}\right|\right] \mathcal{A}_{T},
$$

which corresponds to the amplitude equation near the carrier of frequency $\Omega_{c}=\Omega_{0}-\left(\varpi+\frac{1}{2} \Omega_{T}\right)$. From Eq. (17), we deduce that $\varpi<\Omega_{T}$, so that $\left|\Omega_{c}-\Omega_{0}\right|<\Omega_{T} / 2$ : in case of single-mode oscillation, it means that the oscillating frequency of the microwave $(\sim \mathrm{GHz})$ is detuned by a very small amount $(\sim \mathrm{kHz})$, in all cases smaller than the half free spectral range. Introducing a phase shifter in the loop would therefore enable a fine tuning of the oscillating frequency, but it is not required for the oscillation itself. Equation (18) is therefore the one we are going to use to perform the stability analysis of the system.

\section{EXISTENCE AND STABILITY OF FIXED POINTS}

The stationary states $\mathcal{A}_{s t}$ of Eq. (18) obey the following algebraic equation

$$
\mathcal{A}_{s t}\left\{1-2 \gamma \mathrm{Jc}_{1}\left[2\left|\mathcal{A}_{s t}\right|\right]\right\}=0,
$$

which has two solutions: a trivial solution $\mathcal{A}_{0}=0$, and a non-trivial solution $\mathcal{A}_{\text {osc }}$ obeying $2 \gamma \mathrm{Jc}_{1}\left[2\left|\mathcal{A}_{\text {osc }}\right|\right]=1$. The following subsections are devoted to their stability study, or in other words, to the determination of the parameter range for which these states may be observed.

\section{A. Stability of the trivial solution}

The trivial solution $\mathcal{A}_{0}=0$ exists for all values of $\gamma$. We have to perturb this fixed point and check if the perturbation grows or decays depending of the value of the gain. Neglecting higher order nonlinear terms, a perturbation $\delta \mathcal{A}$ would obey

$$
\delta \dot{\mathcal{A}}=-\mu \delta \mathcal{A}+\mu \gamma \delta \mathcal{A}_{T}
$$

Hence, setting the perturbation of this fixed point to $\delta \mathcal{A}=$ $\delta \mathcal{A}_{0} e^{\left(\lambda_{q}+i \omega_{q}\right) t}$, the real and imaginary parts of the eigenvalue respectively obey the equations

$$
\begin{aligned}
& \lambda_{q}=-\mu+\mu \gamma e^{-\lambda_{q} T} \cos \omega_{q} T \\
& \omega_{q}=-\mu \gamma e^{-\lambda_{q} T} \sin \omega_{q} T .
\end{aligned}
$$

In fact, a bifurcation occurs when the real part $\lambda_{q}$ of the eigenvalue changes its sign (transversality condition): therefore, the transcendental equation (22) can be linearized around $\lambda_{q}=0$. Taking into account $\mu T \gg 1$, we are led to two possible solutions for $\lambda_{q}$ and $\omega_{q}$ :

- Bifurcation for $\gamma>0$

$$
\omega_{q}=0 \text { and } \lambda_{q} \simeq \frac{\gamma-1}{\gamma T}
$$

- Bifurcation for $\gamma<0$

$$
\omega_{q}=\frac{1}{2} \Omega_{T}+\mathcal{O}(\epsilon) \text { and } \lambda_{q} \simeq \frac{\gamma+1}{\gamma T},
$$

From Eqs. (23) and (24), it is found that $\lambda_{q}<0$ for $|\gamma|<1$ and $\lambda_{q}>0$ for $|\gamma|>1$. If we define $\gamma_{t h}=1$ as the threshold value of the oscillations, the trivial fixed point is stable for $|\gamma|<\gamma_{t h}$, and unstable otherwise. Moreover, it also appears that the relevant bifurcation parameter here is $\gamma \cos \omega T \geq 0$ : hence, starting from now, we will restrict our stability study to the case $\gamma \geq 0$, as it captures all the dynamical features of the system. It is understood that the bifurcation scenario for $\gamma<0$ is the same as $\gamma>0$ with frequency detuning $\frac{1}{2} \Omega_{T}$. 


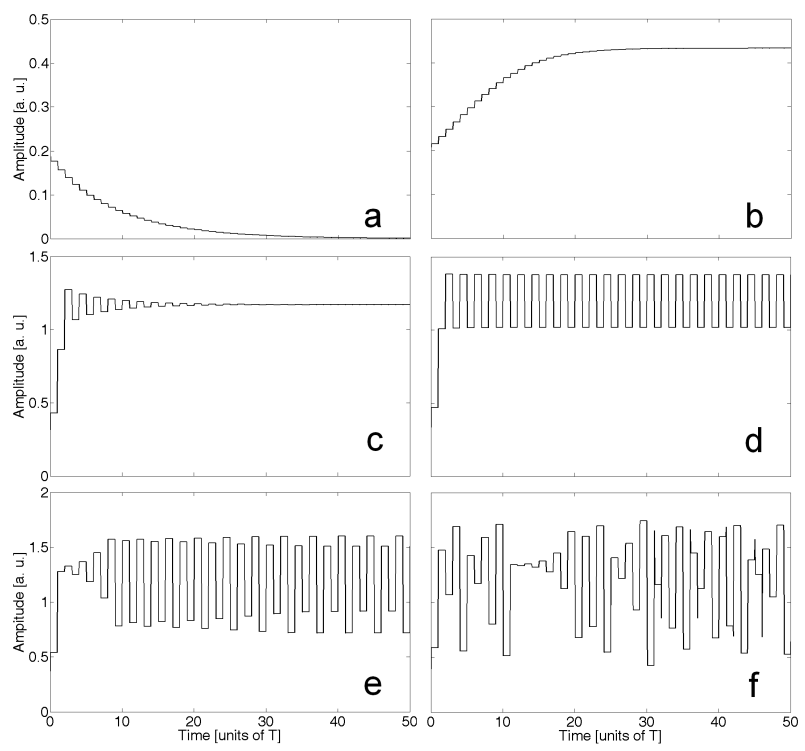

Fig. 2. Numerical simulation of Eq. (18) for various gain values. The initial condition has been uniformly set to $\mathcal{A}=0.2$ for $t \in[-T, 0]$. All the transients are displayed to evidence the winding frequency of the eigenvalues near the bifurcations. a) $\gamma=0.9$; b) $\gamma=1.1$; c) $\gamma=2.2$; d) $\gamma=2.4$; e) $\gamma=2.75$; f) $\gamma=3.0$.

\section{B. Stability of the oscillating solution}

The nontrivial solution is given by

$$
A_{\text {osc }}=\frac{1}{2} \mathrm{Jc}_{1}^{-1}\left[\frac{1}{2 \gamma}\right]
$$

where $\mathrm{Jc}_{1}^{-1}$ is the inverse Bessel-cardinal function, which only exist for $\gamma \geq 1$, and is single-valued for realistic gain values $(\gamma<5)$. In that range, Yao and Maleki have shown the validity of the following approximation (obtained after a fifth-order expansion) [1]

$$
A_{o s c} \simeq \sqrt{3}\left[1-\frac{1}{\sqrt{3}} \sqrt{\frac{4}{\gamma}-1}\right]^{\frac{1}{2}} .
$$

This stationary solution is of particular interest because it corresponds to a pure, single-mode microwave whose frequency $\Omega_{c}$, amplitude $A_{\text {osc }}$ and phase $\psi_{\text {osc }}$ are constant. The stability of this solution depends on the behavior of the amplitude and phase perturbations $\delta A$ and $\delta \psi$, which are governed by the following equations:

$$
\begin{aligned}
\delta \dot{A} & =-\mu \delta A+2 \mu \gamma \mathrm{J}_{1}{ }^{\prime}\left[2 A_{o s c}\right] \delta A_{T} \\
\delta \dot{\psi} & =-\mu\left(\delta \psi-\delta \psi_{T}\right) .
\end{aligned}
$$

It appears that the perturbation equations for the amplitude and the phase are uncoupled, absolutely independent one from each other.

1) Stability of the phase: If we perturb the phase with $\delta \psi=\delta \psi_{0} e^{\left(\lambda_{p}+i \omega_{p}\right) t}$, the real and imaginary parts of the eigenvalue should fulfill the set of equations

$$
\begin{aligned}
& \lambda_{p}=-\mu+\mu e^{-\lambda_{p} T} \cos \omega_{p} T \\
& \omega_{p}=-\mu e^{-\lambda_{p} T} \sin \omega_{p} T
\end{aligned}
$$

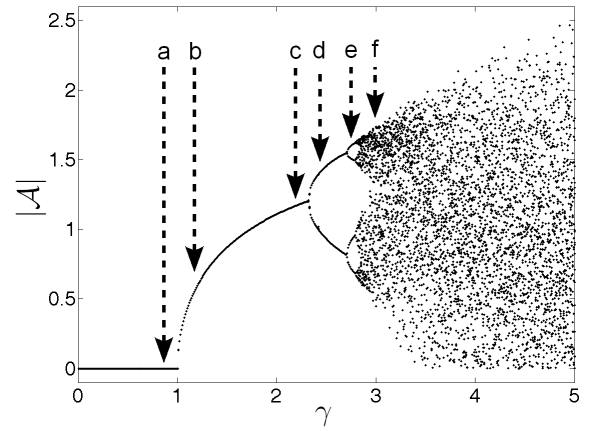

Fig. 3. Numerical bifurcation diagram. The labels a, b, c, d, e and $f$ indicate the dynamical regimes corresponding to the timetraces of Fig. 2.

whose unique physical solution is

$$
\lambda_{p}=0 \text { and } \omega_{p}=0 .
$$

This solution indicates that any value of the phase is allowed and all of them are neutrally stable. This is a direct consequence of the phase invariance associated to limitcycle oscillations.

2) Stability of the amplitude: When we perturb the amplitude of the microwave with $\delta A=\delta A_{0} e^{\left(\lambda_{a}+i \omega_{a}\right) t}$, the real and imaginary parts of the eigenvalue should rather obey

$$
\begin{aligned}
& \lambda_{a}=-\mu+2 \mu \gamma \mathrm{J}_{1}{ }^{\prime}\left[2 A_{o s c}\right] e^{-\lambda_{a} T} \cos \omega_{a} T \\
& \omega_{a}=-2 \mu \gamma \mathrm{J}_{1}{ }^{\prime}\left[2 A_{o s c}\right] e^{-\lambda_{a} T} \sin \omega_{a} T .
\end{aligned}
$$

Close to the bifurcations $\left(\lambda_{a}=0\right)$, the precedent set of equations has two solutions which depend on the sign of the parameter $\alpha=2 \gamma \mathrm{J}_{1}{ }^{\prime}\left[2 A_{o s c}\right]$ :

- Bifurcation for $\alpha>0$

$$
\omega_{a}=0 \text { and } \lambda_{a} \simeq \frac{1-\alpha}{\alpha T},
$$

The bifurcation occurs here at $\alpha=1$, which corresponds to $\gamma=\gamma_{t h}=1$. The damping is such that $\lambda_{a}<0$ for $\gamma>\gamma_{t h}$, while there is simply no oscillating solution for $\gamma<\gamma_{t h}$. Note the continuity of the frequency perturbation at $\gamma_{t h}$, since $\omega_{a}=\omega_{q}=0$.

- Bifurcation for $\alpha<0$

$$
\omega_{a}=\frac{1}{2} \Omega_{T}+\mathcal{O}(\epsilon) \text { and } \lambda_{a} \simeq-\frac{1+\alpha}{\alpha T},
$$

Using Eq. (25) and the equality $\mathrm{J}_{1}{ }^{\prime}(x)=\mathrm{J}_{0}(x)-\mathrm{Jc}_{1}(x)$, it is found that the condition $\alpha=-1$ is fulfilled when the bifurcation occurs at a critical value $\gamma_{c r}$ defined as

$$
\gamma_{c r}=\frac{1}{2 \mathrm{Jc}_{1}\left[\mathrm{~J}_{0}^{-1}(0)\right]} \simeq 2.31
$$

where $\mathrm{J}_{0}^{-1}$ is the inverse of the Bessel-function $\mathrm{J}_{0}$ (in the single-valued range). Here, the damping is such that $\lambda_{a}<0$ for $\gamma<\gamma_{c r}$, and $\lambda_{a}>0$ for $\gamma>\gamma_{c r}$. Therefore, the 


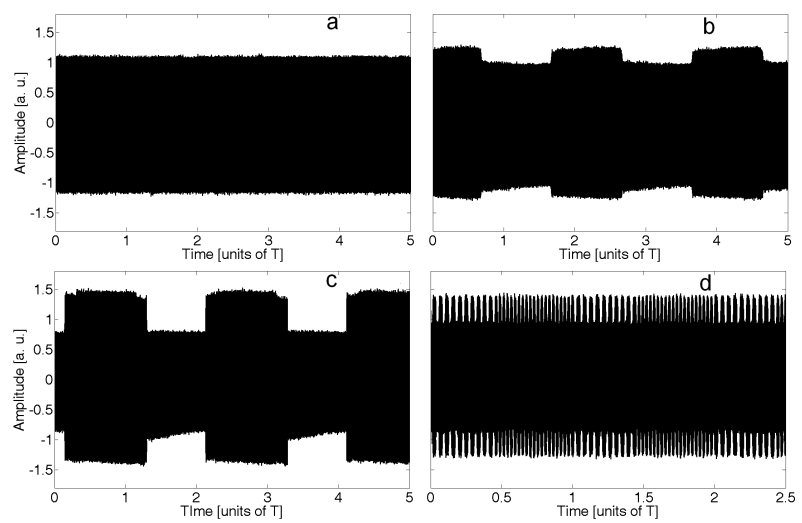

Fig. 4. Experimental results. The carrier has a frequency of $3 \mathrm{GHz}$, and the gain is increased from below to far above the amplitude Hopf bifurcation. a) Just below the critical value, the amplitude is constant; b) Just above the critical value, the amplitude is weakly amplitude modulated; $c$ ) Well above the critical value, the amplitude is strongly amplitude modulated; $d$ ) Far above the critical value, the amplitude modulation becomes very complex, and here it is for example combined to a frequency modulation.

oscillation is stable below the critical value, and unstable above.

From Eqs. (34) and (35), it appears that the stability of the oscillating solution has a lower and an upper boundary as a function of the gain $\gamma$. The lower boundary value is $\gamma_{t h}=1$, and it corresponds to the bifurcation between the trivial and the nontrivial solution since $A_{0}$ is stable for $\gamma<\gamma_{t h}$ while $A_{o s c}$ is stable for $\gamma>\gamma_{t h}$ : this corresponds to a pitchfork bifurcation. Note that here, the negative branch of the "fork" physically corresponds to a positive amplitude with a phase shift of $\pi$. The upper boundary is $\gamma_{c r}=2.31$, and here $A_{o s c}$ is stable as long as $\gamma<\gamma_{c r}$. The winding frequency $\omega_{a}$ of the perturbation does not vanish as $\lambda_{a}$ at the bifurcation point: this is the signature of a Hopf bifurcation, that is, the bifurcation from a fixed point to a limit-cycle of frequency $\omega_{a}$. For $\gamma>\gamma_{c r}$, the microwave oscillation has an amplitude of the form $A_{o s c}+a_{0} \cos \omega_{a} t$, and the theory of Hopf bifurcations shows that the amplitude $a_{0}$ of the modulated component initially grows as $\sqrt{\gamma-\gamma_{c r}}$ beyond the bifurcation.

\section{Numerical Simulations}

The numerical results obtained using Eq. (18) are presented in Fig. 2. Figures $2 \mathrm{a}$ and $2 \mathrm{~b}$ display the transient dynamical behavior of the system just below $(\gamma=0.9)$ and just above $(\gamma=1.1)$ the threshold value $\gamma_{t h}=1$. It can be seen that the amplitude decays to zero or increases to a non-zero stationary value. It can also be noted that the transient behavior is not oscillatory, in agreement with the theoretical stability analysis which had demonstrated that the winding frequency of the eigenvalues near $\gamma_{t h}$ are such that $\omega_{a}=\omega_{q}=0$. Figures $2 \mathrm{c}$ and $2 \mathrm{~d}$ show the amplitude dynamics just below $(\gamma=2.2)$ and just above $(\gamma=2.4)$ the critical bifurcation value $\gamma_{c r}=2.31$. Below the bifurcation, the transients decay with the theoretically predicted winding frequency $\omega_{a}=\frac{1}{2} \Omega_{T}$; however, above the bifurcation, the oscillatory behavior is sustained and the oscillation is steadily amplitude-modulated. Note that the amplitude modulation is a square-wave because the delay is significantly larger than the filter response time $1 / \mu$ (see ref. [10]). As the gain is further increased, the system may enter into a $4 T$-periodic behavior (Fig. 2e), and later into a chaotic behavior (Fig. 2f).

The full bifurcation behavior of the system is shown in the bifurcation diagram of Fig. 3. It synthetically represent the various dynamical states that can be obtained as the feedback gain is varied. This bifurcation sequence to chaos is quite classical, and the Feigenbaum rule may apply: it can therefore be predicted that the $2 T$-periodic solution looses its stability at $\gamma_{f} \simeq \gamma_{c r}+\left(\gamma_{c r}-\gamma_{t h}\right) / \delta=2.6$, where $\delta=4.669 \ldots$ is the Feigenbaum constant. This theoretical value is very near the numerical value of 2.7 obtained from the bifurcation diagram, and because of the very fast convergence of the Feigenbaum sequence, it also nearly correspond to the emergence of chaos.

\section{EXPERIMENTAL RESULTS}

The analytical and numerical analysis is confirmed by the experimental results presented in Fig. 4. The typical sequence from below to above threshold is displayed, and it is evidenced from Fig. 4a to Fig. 4b that the microwave's amplitude effectively undergoes a Hopf bifurcation as it changes from a constant to a steadily periodic behavior of period $2 T$. The experimental value obtained for the critical value was found to be $\tilde{\gamma}_{c r}=2.4 \pm 0.1$, and it has evaluated relatively to the threshold gain.

As it can be seen in Fig. 4c, the modulation depth increases with the gain; furthermore, it is observed that the amplitude modulation can turn asymmetrical. Further increase leads to more complex behaviors: for example, in Fig. 4d, the period of the amplitude modulation drastically drops down to $\sim 1 \mu \mathrm{s}$, and it is at the same time subjected to a frequency modulation of period $T$. This latter regime may be induced by the interaction of several cavity modes, or by higher order integro-differential or nonlinear terms. It also corresponds to the maximum gain that we could achieve experimentally $(\sim 2.7)$, which is sufficient to observe strongly nonlinear regimes, but not enough for fully developed chaos.

\section{Discussion}

From the theoretical, numerical and experimental results, it appears that the behavior of OEOs univocally depends on the value of the normalized gain parameter $\gamma$.

For $\gamma<1$, the systems does not oscillate. From a physical point of view, it can be considered that $\gamma_{t h}=1$ is a threshold value below which the system does not have enough energy to sustain an oscillation, as the energy input is still inferior to the energy losses in the feedback loop. Above the threshold value, the system oscillates and the output is a perfectly pure microwave of frequency $\Omega_{c} \simeq \Omega_{0}$ and arbitrary (but constant) phase, and this regime is stable as long as the gain is inferior to the critical value $\gamma_{c r}=2.31$. Hence, the interval of gain values 
between $\gamma_{t h}$ and $\gamma_{c r}$ induces the single-mode behavior required for OEOs technological applications. For $\gamma>\gamma_{c r}$, the monomode solution becomes unstable, as its amplitude becomes modulated: the modulation depth increases with the gain offset, while the period of the modulation is fixed to twice the time delay. The consequence of this amplitude modulation of the microwave is that in the RF Fourier spectrum, two robust modulation side-peaks appear at $\Omega_{c}-\frac{1}{2} \Omega_{T}$ and $\Omega_{c}+\frac{1}{2} \Omega_{T}$. It is expected that the emergence of these peaks may be detrimental to the phase noise performance of OEOs. In our case for example, when the emitted microwave is stable, there are spurious (noiseinduced) cavity-mode situated at $\pm k \times 50 \mathrm{kHz}$ from the carrier ( $k$ being a strictly positive integer). However, after the loss of stability, additional deterministic side-peaks also appear at $\pm 25 \mathrm{kHz}$ [14]. This phenomenology is completely deterministic, that is, it does not correspond to an hidden influence of noise in the system: it is a bifurcation behavior induced by the nonlinear function $\mathrm{J}_{1}$ which is ruling the dynamics of the envelope amplitude.

It is noteworthy that as long as the bandwidth $\Delta \Omega=2 \mu$ is significantly smaller than the oscillation frequency $\Omega_{c} \simeq$ $\Omega_{0}$, it does not play any role in this bifurcation sequence. Effectively, the stationary amplitude, as well as the critical bifurcation values $\gamma_{t h}$ and $\gamma_{c r}$ are independent of $\mu$. This is also a proof that in first approximation, the exact profile of the bandpass filter is not a critical parameter: the same results would have been obtained with a filter whose edges are steeper $i$. e., a filter whose falling edges decrease faster than $-20 \mathrm{~dB} / \mathrm{dec}$, as it is likely to be the case for a real world RF filter. It should also be emphasized that reducing the bandwidth of the $\mathrm{RF}$ filter can not prevent from the microwave's loss of stability beyond $\gamma_{c r}$, except for $\Delta \Omega / 2 \pi \ll \Omega_{T} / 2 \pi=50 \mathrm{kHz}$ (bandwidth smaller than the free spectral range). Unfortunately, there are actually no available RF filters with such a narrow bandwidth, so that experimentally the Hopf bifurcation at $\gamma_{c r}$ can by no way be circumvented through ultra-selective filtering.

The existence of a maximum gain value $\gamma_{c r}$ for the monomode oscillation has another important consequence as far as applications are concerned: according to Eq. (25), the maximal output amplitude of the monomode microwave is $A_{\max }=\frac{1}{2} \mathrm{Jc}_{1}^{-1}\left[\frac{1}{2 \gamma_{c r}}\right]=1.20$. Hence, the maximum value of the root mean square voltage at the MachZehnder input is

$$
V_{r m s_{\max }}=\frac{A_{\max }}{\sqrt{2}} \frac{2 V_{\pi_{R F}}}{\pi} \simeq 0.54 \times V_{\pi_{R F}},
$$

that is, about half the value of $V_{\pi_{R F}}$ : The maximum microwave output power is therefore of the order of $100 \mathrm{~mW}$ (or $20 \mathrm{dBm}$ ) under a $50 \Omega$ impedance. In our case, it corresponds to peak to peak voltage $V_{p p_{\max }} \sim 6 \mathrm{~V}$, which is smaller than the $12 \mathrm{~V}$ limit of our driver, thereby enabling the observation of instabilities. This gives a direct indication about the maximum microwave output power that can be extracted from a single-mode OEO. At last, it should be reminded that the phase noise floor decreases in an inversely proportional way relatively to the microwave power: therefore, the limitation induced by $\gamma_{c r}$ also defines a limitation for the power-induced downshift. A higher microwave power may naturally be obtained through postamplification, at the expense of phase noise performance, though.

From a mathematical point of view, the envelope equation (18) is a member of the Ikeda equations family [17]. Its stability analysis has revealed that the phase does not play a role for $\gamma<1$ (no oscillations), and does not either play a role for $1<\gamma<2.31$ (neutrally stable phase). Therefore, for $\gamma<\gamma_{c r}$, the complex envelope equation can be reduced to a real equation, exclusively ruling the amplitude dynamics:

$$
\dot{A}=-\mu A+\mu \gamma \mathrm{J}_{1}\left[2 A_{T}\right] .
$$

This equation is similar to the real Ikeda equation with the difference that the nonlinear function here is a Bessel function instead of a sinusoidal one. The advantage of using the real equation (38) is that the theoretical analysis is simpler and the numerical simulations are speeded up by a factor 2. The drawbacks are that this equation is only valid below the Hopf bifurcation, and it does not anymore carry any information about the phase, so that it can not be used later for phase noise studies.

At last, the integro-differential delay equation (4) ruling the dynamics of the initial microwave variable $x(t)$ is also an Ikeda-like equation [9], and the bifurcation phenomena occurring for the $\mathcal{A}$ variable can also be interpreted relatively to $x$. Then, below $\gamma_{t h}=1$, the solution $x(t) \equiv 0$ is stable. Above the threshold value, an monomode oscillation of frequency $\Omega_{c}$ emerges through a Hopf bifurcation, as $x$ switches from a fixed point to a limit-cycle. At $\gamma_{c r}=2.31$, as the amplitude $A$ becomes modulated, the limit-cycle corresponding to $x(t)$ bifurcates to a torus: this is a secondary Hopf bifurcation, also called Neimark-Sacker bifurcation.

\section{CONCLUSION}

In this article, we studied the nonlinear dynamics of optoelectronic oscillators. We have first shown that the microwave amplitude obeys a real integro-differential equation with delay. Then, using a multiple time scale analysis, we have derived a reduced nonlinear delay-differential equation whose variable is the complex slowly-varying envelope of the microwave.

The stability analysis of the equation has been performed, and it has been shown that as the gain is increased beyond a given critical value, the amplitude of the oscillations undergoes a supercritical Hopf bifurcation. This bifurcation induces an amplitude modulation whose principal consequence is the appearance of deterministic side-peaks around the carrier, thereby deteriorating the spectral purity of the oscillations. Numerical simulations and experimental measures have confirmed the theoretical analysis, as all the bifurcation phenomena have effectively been observed following the predicted scenario.

This bifurcation has important implication for the various applications of these oscillators: in particular, it sets 
an upper limit to the output power of an ultra-pure microwave. However, one can expected that conventional methods of bifurcation control should at least theoretically enable to shift that critical bifurcation value if necessary.

An interesting extension of the amplitude equation introduced here would be the inclusion of noise terms in the amplitude equation allowing for the study the phase noise in OEOs (Langevin formalism). Effectively, time domain noise analysis is quite scarcely used in phase studies because there is generally no deterministic equation to rule the phase dynamics. For the OEO, the use of our complex envelope equation will permit to apply the formalism of stochastic differential equations in order to quantify and analyze the effect of random fluctuations on the phase noise performances of optoelectronic oscillators.

\section{REFERENCES}

[1] X. S. Yao and L. Maleki, "Optoelectronic microwave oscillator", J. Opt. Soc. Am. B 13, pp. 1725-1735 (1996).

[2] X. S. Yao and L. Maleki, "Optoelectronic oscillator for photonic systems", IEEE J. of Quantum Electron. 32, pp. 11411149 (1996)

[3] X. S. Yao and L. Maleki, "Multiloop optoelectronic oscillator", IEEE J. of Quantum Electron., 36, pp. 79-84 (2000).

[4] E. Salik, N. Yu and L. Maleki, "An Ultralow Phase Noise Coupled Optoelectronic Oscillator", IEEE Photon. Technol. Lett., 19, pp. 444-446 (2007).

[5] S. Poinsot, H. Porte, J.-P. Goedgebuer, W. T. Rhodes and B. Boussert, "Continuous radio-frequency tuning of an optoelectronic oscillator with dispersive feedback", Opt. Lett. 27, 13001302 (2002);

[6] D. Strekalov, D. Aveline, N. Yu, R. Thompson, A. B. Matsko and L. Maleki, "Stabilizing an optoelectronic microwave oscillator with photonic filters", J. Lightwave Technol. 21, 3052-3061 (2003).

[7] J. Lasri, P. Devgan, R. Tang and P. Kumar, "Ultralow timing jitter $40-\mathrm{Gb} / \mathrm{s}$ clock recovery using a self-starting optoelectronic oscillator", IEEE Photon. Technol. Lett. 16, 263-265 (2004).

[8] A. Neyer and E. Voges, "Dynamics of electrooptic bistable devices with delayed feedback", IEEE J. of Quantum Electron., 18, pp. 2009-2015 (1982).

[9] Y. Chembo Kouomou P. Colet, L. Larger and N. Gastaud, "Chaotic breathers in delayed electro-optical systems", Phys. Rev. Lett. 95, 203903-1-4 (2005).

[10] T. Erneux, L. Larger, M. W. Lee and J.-P. Goedgebuer "Ikeda Hopf bifurcation revisited", Physica D 194, 49-64 (2004).

[11] N. Gastaud, S. Poinsot, L. Larger, J.-M. Merolla, M. Hanna, J.-P. Goedgebuer and F. Malassenet, "Electro-optical chaos for multi-10 Gbit/s optical transmissions", Electron. Lett. 40, 898899 (2004).

[12] Y. Chembo Kouomou, P. Colet, L. Larger and N. Gastaud, "Mismatch-induced bit error rate in optical chaos communications using semiconductor lasers with electrooptical feedback", IEEE J. of Quantum Electron., 41, pp. 156-163 (2005).

[13] A. Argyris, D. Syvridis, L. Larger, V. Annovazzi-Lodi, P. Colet, I. Fischer, J. García-Ojalvo, C. R. Mirasso, L. Pesquera and K. A. Shore, "Chaos-based communications at high bit rates using commercial fibre-optic links", Nature 438, 343-346 (2005).

[14] Y. Kouomou Chembo, L. Larger, H. Tavernier, R. Bendoula, E. Rubiola and P. Colet "Dynamic instabilities of microwaves generated with optoelectronic oscillators", Opt. Lett. 32, 25712573 (2007)

[15] Ali H. Nayfeh, "Introduction to perturbation techniques", Wiley-Interscience, New-York (1981).

[16] Y. Chembo Kouomou and P. Woafo "Triple resonant states and chaos control in an electrostatic transducer with two outputs", J. Sound Vib. 270, 75-92 (2004).

[17] K. Ikeda, "Multiple-valued stationary state and its instability of the transmitted light by a ring cavity system", Opt. Commun. 30, 257-261 (1979). 